\title{
End points in asthma
}

$\mathrm{T}$ his issue of the Canadian Respiratory Journal contains an unusual paper. Parameswaran et al (1) discuss, in detail, methods to compare the effects of different inhaled corticosteroids in asthma. Few specific data are presented comparing one molecule with another, but various approaches to such comparisons are reviewed. The message is that it is not easy to make such comparisons. This is the case because, to a large extent, steroid dose-response curves in asthmatic patients tend to be flat; that is, a large benefit is frequently seen at a relatively low dose of any inhaled steroid, and higher doses produce little added benefit. Thus, comparing steroid drugs requires studies using unusually low doses of the relevant agents - doses that would likely not be used clinically. Parameswaran et al point out that this is especially true for the commonly used measures of lung function (forced expiratory volume in $1 \mathrm{~s}\left[\mathrm{FEV}_{1}\right]$, peak expiratory flow $[\mathrm{PEF}]$ ) that are used to assess asthmatic patients. They also explain that most asthmatic patients have normal or near normal values of $\mathrm{FEV}_{1}$ and $\mathrm{PEF}$, and are therefore unlikely to improve very much with more therapy of any type. Alternative end points may be more sensitive in relatively well controlled asthmatic patients. These include measures of airway reactivity in response to allergens or other inhaled agents. Surrogate measures of airway inflammation such as exhaled nitric oxide or sputum eosinophils could be used. Unfortunately, the evidence that these techniques have a clear steroid dose-response effect is not undisputed, and they are probably sensitive to the duration of steroid therapy as well as the dose. Most of these approaches also require special expertise and/or equipment.

A more attractive idea is to compare inhaled steroids in asthmatic patients who are not well controlled, but again, we run into the dose-response problem, and relatively few patients are so poorly controlled that major improvements in lung function would be likely. Even better, from the scientific point of view, would be to take patients who are satisfactorily controlled on a relatively high dose of inhaled steroid therapy and reduce the dose, with loss of control as the end point. This protocol could be repeated for a number of agents. Obviously, the patients would have to be followed up very carefully, and would have to be very familiar with appropriate rescue protocols. This is the model that Parameswaran et al regard as the most promising.

Parameswaran et al point out that doses of inhaled agents depend on the delivery system, including items such as the particle size generated and the presence or absence of a spacer. Doses can be estimated in terms of fine particles or fractions of the nominal dose deposited in the lungs. Again, these measurements involve specialized techniques.
Many of the issues raised by Parameswaran et al revolve around what is important to measure in asthmatic patients or what simple, reproducible end point is of true clinical significance. That is, what should we use as an index of benefit in patients with asthma? I believe that this is a simpler, and more important, issue than how to compare different inhaled

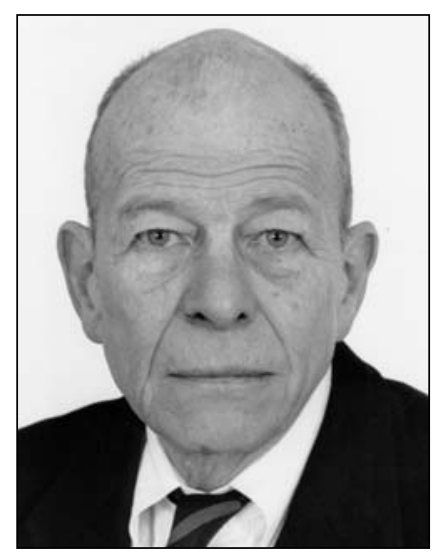

Nick R Anthonisen corticosteroids. As noted above, most asthmatic patients do not have clear-cut abnormalities of lung function to try to improve. Given this, I think that the most important item to consider is whether the disease is under control. Lack of control implies compromised quality of life and increased health care expenditures. 'Control' may be defined in a number of ways. The most obvious are emergency room visits, hospitalizations and courses of prednisone. These are serious events but may not be very common among most asthmatic patients. More sensitive measures of control are responses to symptom questionnaires, and daily records of PEF and the use of short acting betaagonists. I was recently involved in a randomized trial of the influenza vaccine in some 2000 American asthmatic patients (2) who were recruited off of the street and who were generally not very sick at the time of vaccination. On the other hand, many of them were under relatively poor control in terms of their symptoms and beta-agonist use, and in the fourweek observation period associated with the study, there was a surprisingly high rate of significant deterioration, up to $40 \%$. It appears that many asthmatic patients are not well controlled, that this is a robust and easy end point to measure, and finally, that it is of clear clinical significance.

Nick R Anthonisen MD Editor-in-Chief Canadian Respiratory Journal

\section{REFERENCES}

1. Parameswaran K, Leigh R, O’Byrne PM, Kelly MM, Goldsmith CH, Hargreave FE, Dolovich M. Clinical models to compare the safety and efficacy of inhaled corticosteroids in patients with asthma. Can Respir J 2003;10:27-34.

2. American Lung Association Asthma Clinical Trials Research Centers. The safety of inactivated influenza vaccine in adults and children with asthma. N Engl J Med 2001;345:1529-36. 


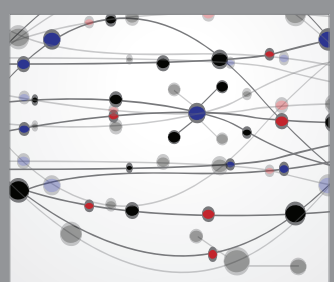

The Scientific World Journal
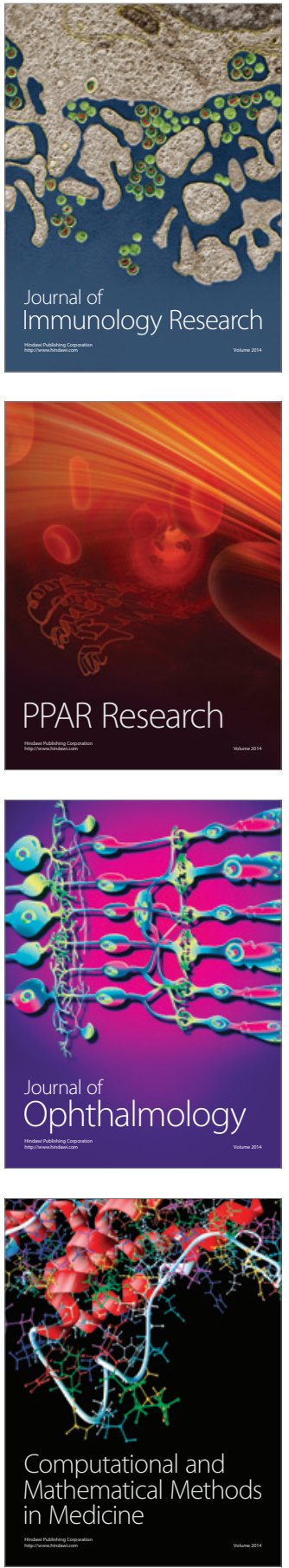

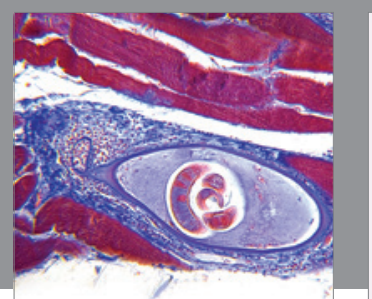

Gastroenterology Research and Practice

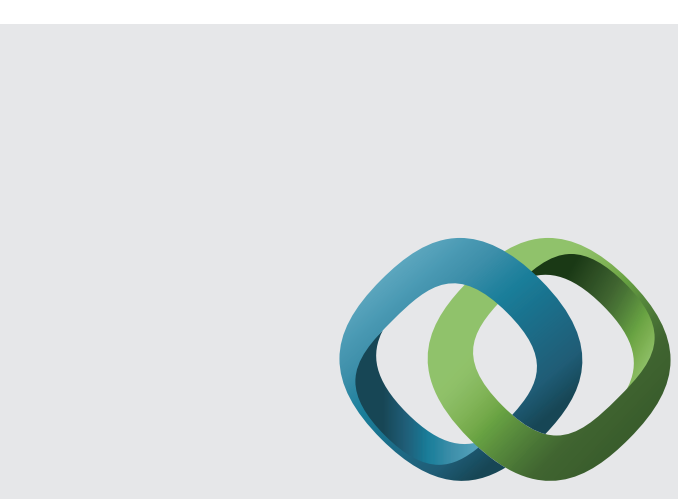

\section{Hindawi}

Submit your manuscripts at

http://www.hindawi.com
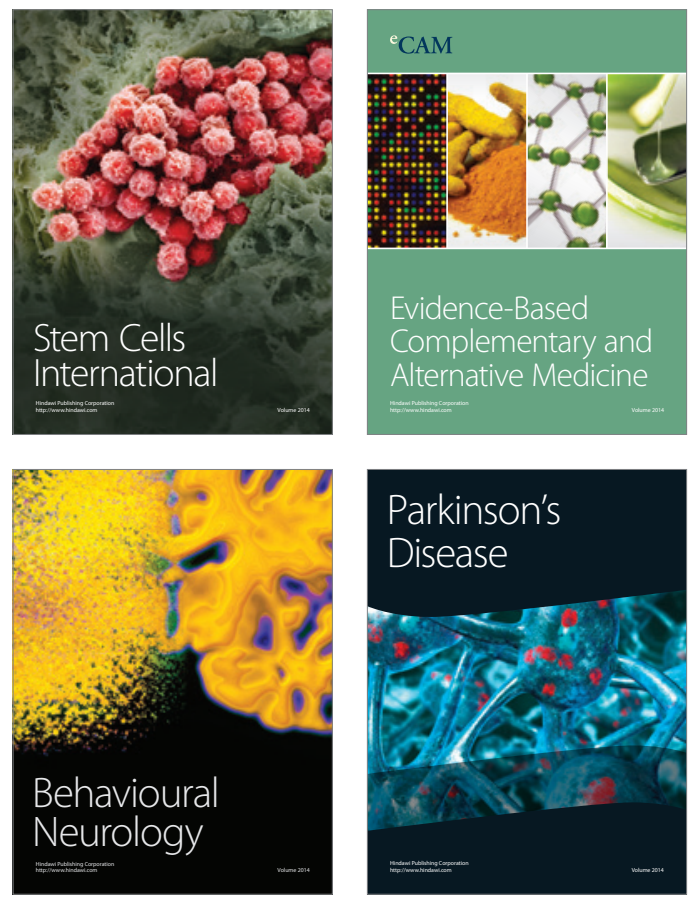
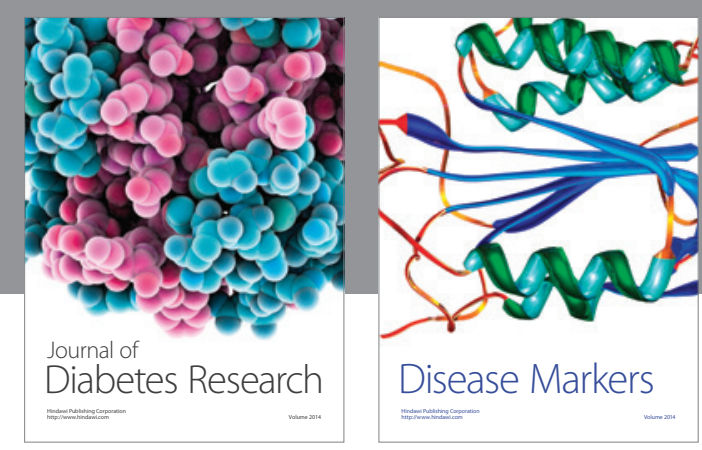

Disease Markers
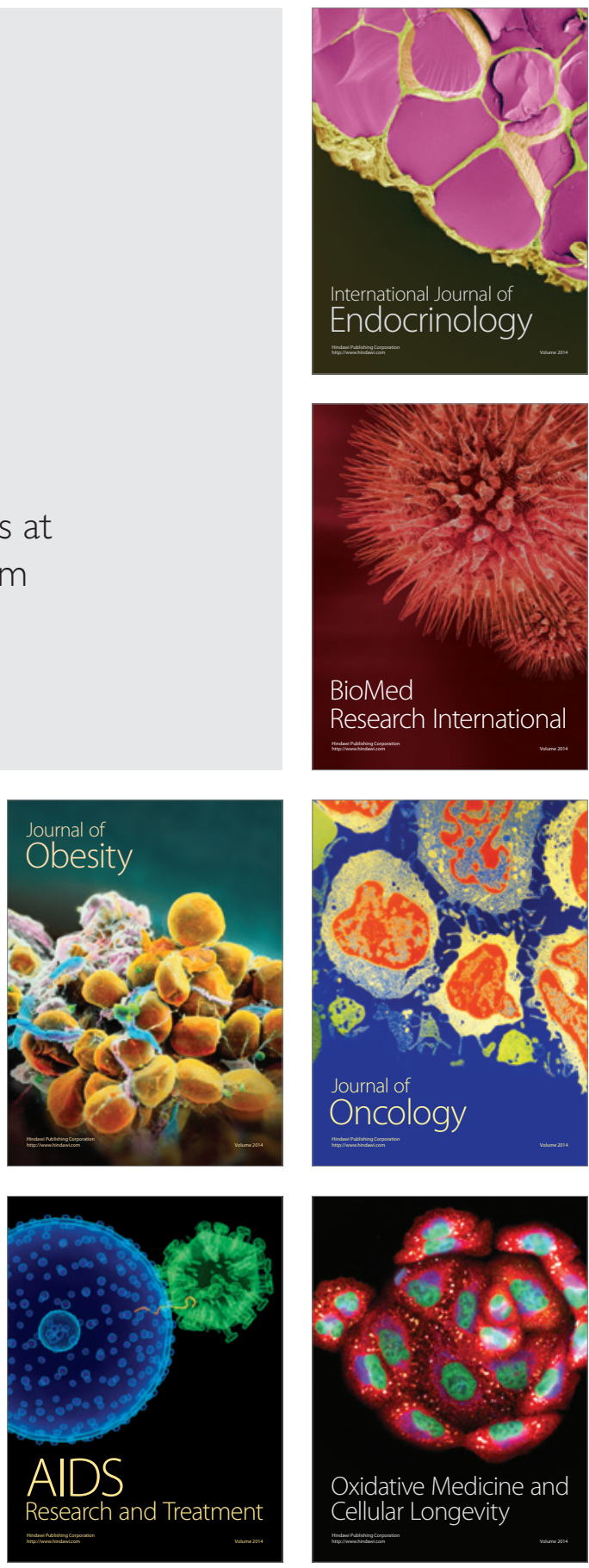